\title{
Diagnostic accuracy of sFlt1/PIGF ratio as a marker for preeclampsia
}

\author{
Pooneh Nikuei ${ }^{{ }^{*}}$, Minoo Rajaei ${ }^{1}$, Nasibeh Roozbeh ${ }^{2}$, Fatemeh Mohseni ${ }^{3}$, Fatemeh Poordarvishi ${ }^{3}$, Mohsen Azad ${ }^{4}$ and \\ Solmaz Haidari ${ }^{1}$
}

\begin{abstract}
Background: Preeclampsia (PE) is a serious complication of pregnancy and one of the main causes of maternal and neonatal mortality and morbidity in the world. Finding a biomarker with high sensitivity and specificity could lead to prediction and early diagnosis of the disease and reduces its complications. In this study, we evaluated diagnostic accuracy of Soluble fms-like tyrosine kinase-1 (sFlt-1) to Placental growth factor (PIGF) ratio for diagnosis of PE.
\end{abstract}

Methods: The cases included 23 mild, 15 severe preeclamptic patients, and 20 normal term pregnant women as control referred to GYN ward of the Persian Gulf Hospital in Bandar Abbas from 2014 to 2016. Levels of sFlt-1 and PIGF were measured. Receiver Operating Characteristic (ROC) curve analysis was applied to calculate diagnostic accuracy of sFlt-1/PIGF ratio.

Results: The mean Level of sFlt-1/PIGF in PE patients $(91.33 \mathrm{ng} / \mathrm{ml})$ was significantly higher than control women (17.62) $(P<0.001)$. ROC curve analysis showed sFIt-1/PIGF ratio diagnostic accuracy in preeclamptic patients with Area Under Curve (AUC) of 0.90 , the best cut-off value of 24.96 , sensitivity and specificity of 84.2 and $85.0 \%$, respectively.

Conclusions: Our data showed sFlt-1/PIGF ratio has higher accuracy for differentiating PE patients from non-PEs in comparison with its power for differentiating severe or early onset forms of the disease.

\section{Background}

Preeclampsia (PE) is diagnosed after 20 weeks of gestation [1]. This multisystem disorder affects $2-7 \%$ of pregnant women [2]. PE has serious complications including liver and kidney dysfunction, seizure and even death. PE is one of the major causes of maternal death, neonatal mortality and premature deliveries [3, 4]. As long as the placenta is present, PE is likely to happen and the recovery process starts post-delivery following the removal of placenta [5]. PE is divided into early-onset and lateonset according to the time of onset. Early-onset PE develops before 34 weeks of gestation, while late-onset $\mathrm{PE}$ develops at or after 34 weeks of gestation. The etiology of early-onset form of $\mathrm{PE}$ is related to incomplete trophoblast invasion and failure of normal spiral artery remodeling. Late-onset PE is associated with increased

\footnotetext{
* Correspondence: Pooneh.Nikuei@hums.ac.ir

${ }^{1}$ Fertility and Infertility Research Center, Hormozgan University of Medical

Sciences, Bandar Abbas, Iran

Full list of author information is available at the end of the article
}

maternal vascular susceptibility to the normal inflammatory state of pregnancy or atherosis of a placenta grown initially normal [6]. Diagnostic criteria for PE include hypertension after 20 weeks of gestation and the coexistence of one or more of the following new-onset conditions: proteinuria, maternal organ dysfunction, and uteroplacental dysfunction [7]. Definite pathogenesis of $\mathrm{PE}$ is not completely clear, but the imbalance between angiogenic factors like vascular endothelial growth factor or placental growth factor (PlGF) and anti-angiogenic factors like soluble fms-like tyrosine kinase 1 (sFlt-1) are known to be related to the disease pathogenesis $[8,9]$.

In normal pregnancies, the level of sFLT-1 starts to rise after 30-32 weeks of gestation and PlGF level starts to decrease after 30 weeks of gestation. Actually cellular stress in the syncytiotrophoblast, which occurs during the last 8-10 weeks of a pregnancy leads to biochemical changes in levels of sFlt-1 and PIGF in normal pregnancies [10]. Circulating levels of sFlt-1 and PlGF alter in PE patients. This alteration begins before the disease onset and stays 
during the course of the disease [11]. In women with PE sFlt-1 rises approximately 5 weeks prior to disease onset [12] while the level of PlGF decreases before the rising of sFlt-1 [12, 13]. Hence, to improve the quality of PE diagnosis, some studies suggest sFlt1/PlGF Ratio as a better marker compared to measuring sFlt1 or PlGF separately for diagnosis of PE [11]. The aim of this study was to evaluate the diagnostic accuracy of sFlt-1: PlGF ratio, and the simplified best cut-off values for sFlt-1:PlGF ratio in assessing the diagnosis of PE.

\section{Methods}

This research was a case-control study. Subjects were chosen from pregnant women referred to the Persian Gulf hospital located in Bandar Abbas in the south of Iran from 2014 to 2016. There were $38 \mathrm{PE}$ patients and 20 normal term pregnant women as controls in the study. Informed written consent was obtained from all participants according to the protocols approved by the Ethical Committee of Hormozgan University of Medical Sciences (No. 1-HEC-93-7-8). Patients were subgrouped as mild (23 women) and sever (15 women). Patients were classified into early-onset ( 9 women) and late-onset ( 29 women). Patients with diabetes, renal disease, collagen vascular diseases, and chronic hypertension were excluded from the study. PE was defined as gestational hypertension (systolic pressure $>140 \mathrm{mmHg}$ or diastolic blood pressure $>90 \mathrm{mmHg}$ on two or more occasions after gestational week 20) with proteinuria (> $0.3 \mathrm{~g} /$ day). The disease was defined severe if, more than one of the following criteria were met: (i) severe gestational hypertension; that is, systolic pressure $>160$ $\mathrm{mmHg}$ or diastolic blood pressure $>110 \mathrm{mmHg}$ on two or more occasions after gestational week 20, (ii) severe proteinuria; that is, protein $\geq 5 \mathrm{~g}$ in a 24 -h urine specimen, (iii) oliguria, (iv) cerebral or visual disturbances, (v) pulmonary edema or cyanosis, (vi) epigastric or right upper quadrant pain, (vii) impaired liver function, (viii) thrombocytopenia or (ix) fetal growth restriction [1]. PE was defined early-onset $(<34$ weeks of gestation) and late-onset ( $\geq 34$ weeks) [14]. The whole blood samples were obtained from participants and centrifuged at 3000 $\mathrm{g}$ for $20 \mathrm{~min}$ and separated serum samples stored at $80^{\circ} \mathrm{C}$ for ELISA. Levels ofsFlt- 1 and PIGF were measured using (ELISA, R\&D System, Human VEGF R1/Flt1Immunoassay, Catalog Number DVR100B and Human PIGF Immunoassay, Catalog NumberDPG00) according to the procedure provided by the manufacturer.

\section{Statistical analysis}

One-way ANOVA/Kruskal-Wallis test with Bonferroni correction was applied for comparison of data between multiple groups and Mann-Whitney test / unpaired ttest was used for comparison between two groups based on the normality of data. Data were shown as number $(\%)$, mean $( \pm \mathrm{SD})$. The Receiver Operating Characteristics (ROC) curve analysis was applied to calculate the Area Under the Curve (AUC) and to find the best cut-off point, Positive Predictive Values (PPVs), Negative Predictive Values (NPVs), diagnostic accuracy, sensitivity, specificity and likelihood ratios.

\section{Results}

We did not observe any significant difference in maternal age $(P=0.546)$ and BMI before pregnancy $(P=0.355)$ between case and control groups. This showed that case and control groups were matched. Clinical characteristics are displayed in Table 1. The number of nulliparous women in both groups was almost similar $(40 \%$ in the control group and $44.7 \%$ in the case group). Recurrent PE was observed in $15.8 \%$ of women in the case group.

Mean Level of sFlt-1/PlGF was $91.33 \mathrm{ng} / \mathrm{ml}$ in PE patients and 17.62 in controls which were increased significantly $(P<0.001)$. In comparison between mild, severe and controls the mean level of sFlt-1/PlGF was 130.97 in severe $P E$ patients which was significantly increased compared to controls $(P<0.001)$. Also, it was 65.48 in mild PE patients which was increased significantly compared to controls $(P<0.001)$. Although the level of sFlt-1/PlGF was higher in severe PE patients compared to mild women, there was no significant difference between these two groups $(P=0.389)$. Also in the comparison between earlyonset, late-onset and controls the mean level of sFlt-1/ PlGF was 153.44 in the early-onset PE patients and 72.06 in late-onset PE patients. There was a significant difference between early-onset group and controls $(P<0.001)$, and also between late-onset PE patients and controls $(P<0.001)$, but there was no significant difference between early and late onset PE patients (0.503) (Fig. 1).

\section{ROC-curve analysis}

The ROC curve analysis was applied to differentiate PE patients from normal controls and also for differentiating severe and early-onset forms of PE using sFlt-1/PIGF ratio. The results for differentiation of PE patients from normal pregnancies showed an AUC of 0.90 (95\% CI $=0.83-0.98$ ). The best cut-off value was $24.96 \mathrm{ng} / \mathrm{ml}$ with sensitivity of $84.2 \%(95 \% \mathrm{CI}=68.7-94)$ and specificity $85 \%(95 \% \mathrm{CI}=$ 62.1-96.8). Table 2 and Fig. 2 provide results related to LR, PPV, and NPV. Moreover, ROC curve analysis was also applied to differentiate early onset PE from controls using sFlt-1/PIGF ratio (Table 2 and Fig. 2).

\section{Discussion}

Anti-angiogenic factors like sFlt-1 are released by the placenta in the circulation of women affected to PE and are known as the cause of endothelial dysfunction 
Table 1 Clinical characteristics of the participants

\begin{tabular}{llll}
\hline Characteristics & PE (38) & Controls (20) & $P$-value \\
\hline Maternal age (year) & $27.18 \pm 5.86$ & $26.25 \pm 4.93$ & 0.546 \\
Placental weight(g) & $423.95 \pm 87.37$ & $613.50 \pm 53.44$ & $<0.001^{*}$ \\
BMI before pregnancy $(\mathrm{kg} / \mathrm{m} 2)$ & $23.81 \pm 3.98$ & $23.21 \pm 3.50$ & 0.355 \\
Systolic Blood Pressure $(\mathrm{mmHg})$ & $150.68 \pm 1.61$ & $110.23 \pm 0.70$ & $<0.001^{*}$ \\
Diastolic Blood Pressure $(\mathrm{mmHg})$ & $100.07 \pm 0.95$ & $60.70 \pm 0.66$ & $<0.001^{*}$ \\
\hline
\end{tabular}

Results are presented as mean \pm SD. $P<0.05$ is considered significant

observed in this disease. This anti-angiogenic protein inhibits the activity of VEGF and PIGF and results in hypertension and proteinuria as main symptoms of $\mathrm{PE}$ [11]. Recombinant sFlt-1 could block development of endothelial tubes and inhibit vasodilatory effects of VEGF and PIGF in vasculature [15]. In women with PE, the levels of sFlt- 1 rise 5 weeks prior to clinical presentations, and stay elevated till the onset of the disease [12,
16] while the serum PIGF decreases before clinical presentation [12]. So these two markers could be used for prediction and diagnosis of $\mathrm{PE}$, but recent studies showed that sFlt1/PIGF ratio is a better marker with more accuracy for screening PE rather than sFlt-1 or PlGF alone [17].

According to our knowledge, this is the first study which investigates the diagnostic accuracy of sFlt1/PlGF
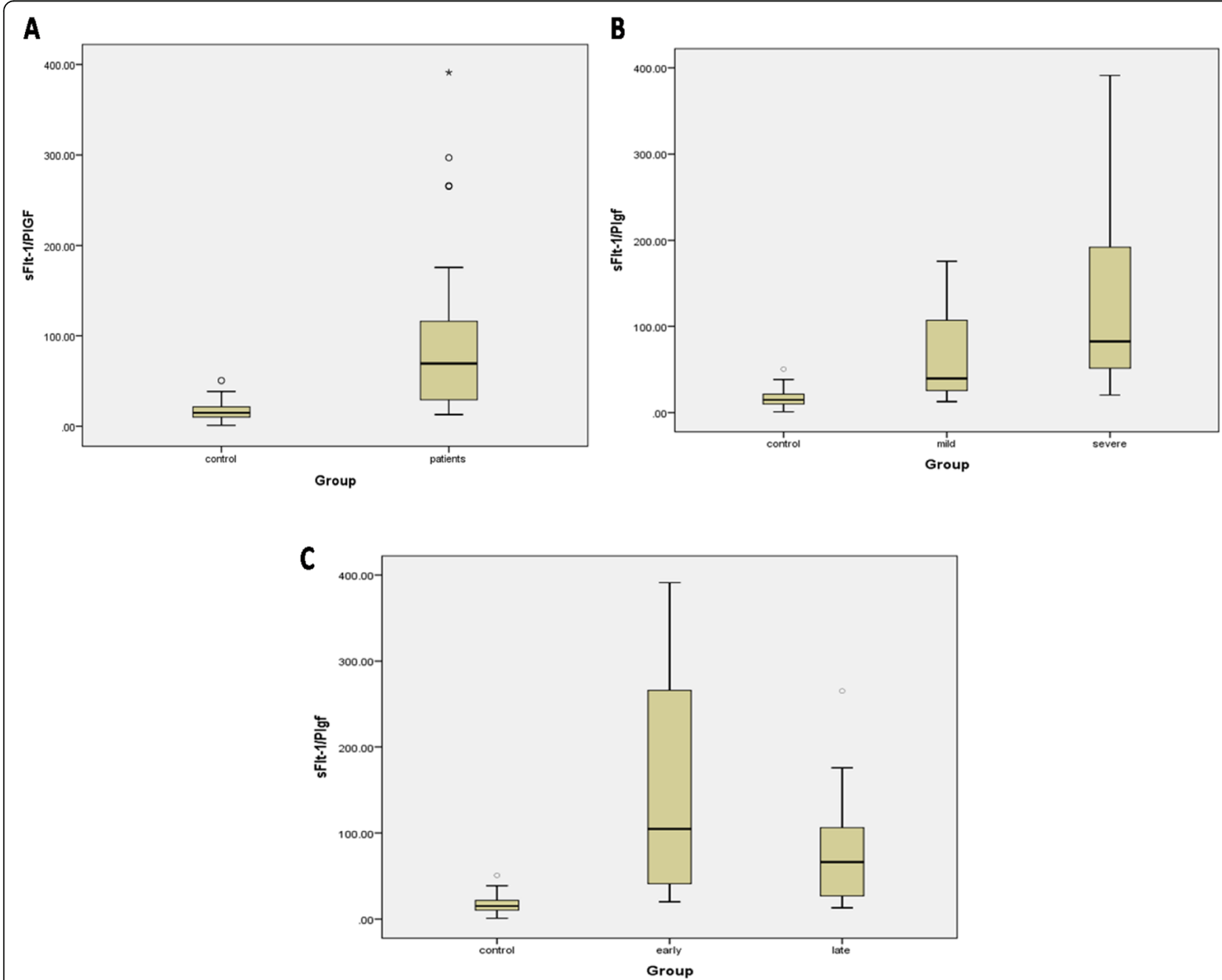

Fig. 1 a Comparison of sFlt-1/PIGFratio between PE patients and controls; $\mathbf{b}$ mild and sever PE patients with controls; $\mathbf{c}$ early and late onset PE patients with controls, which shows significant increase in all PE groups in comparison with the normal women. The 10th and 90th centiles are represented by lower and upper bars. The horizontal line in the box shows median 
Table 2 Diagnostic accuracy of sFlt/PIGF ratio for diagnosing preeclampsia

\begin{tabular}{lllllllll}
\hline Groups & cut off & Sensitivity & Specificity & LR+ & LR- & PPV & NPV & Area (95\% Cl) \\
\hline PE & 24.96 & $84.2(68.7-94)$ & $85(62.1-96.8)$ & 5.61 & 0.186 & 91.4 & 73.9 & $90(0.83-0.98)$ \\
Sever PE & 40.9 & $80(51.9-95.7)$ & $72.09(56.3-84.7)$ & 2.8 & 0.27 & 50 & 91.2 & $0.80(0.65-0.95)$ \\
Early-onset PE & 39.7 & $88.89(51.8-99.7)$ & $65.31(50.4-78.3)$ & 2.56 & 0.17 & 32 & 97 & $0.80(0.65-0.95)$ \\
\hline
\end{tabular}

LR Likelihood Ratio, PPV Positive Predictive Value, NPV Negative Predictive Value, CI Confidence Interval

ratio in Iranian women with PE. Results of our study showed sFlt1/PlGF ratio has the highest accuracy for differentiating PE patients from normal pregnant women. In our study the best cut-off for the highest sensitivity (84.2\%) and specificity (85\%) was $24.96 \mathrm{ng} / \mathrm{ml}$ with $\mathrm{LR}^{+}$ 5.61, LR $^{-} 0.186$ for differentiating PE from healthy women. So because of high sensitivity, specificity, positive and negative LR of sFlt1/PlGF ratio, it could help in diagnosing high-risk women for developing PE and decreasing costs related to serious maternal and neonatal complications by precise observation and early medical interventions. Also, we evaluated the accuracy of sFlt1/ PIGF ratio in diagnosing severe and early-onset PE which more likely lead to preterm delivery.

Based on our ROC curve analysis for sFlt1/PlGF ratio, the best cut-off for diagnosing severe PE patients is 40.9 $\mathrm{ng} / \mathrm{ml}$ with $80 \%$ sensitivity and $72.09 \%$ specificity, and the best cut-off for diagnosing early-onset PE is $39.7 \mathrm{ng} / \mathrm{ml}$ with sensitivity of $88.89 \%$ and specificity of $65.31 \%$. In this study, sFlt1/PlGF ratio showed the highest sensitivity and specificity in differentiating PE patients from normal pregnant women. Our results are in agreement with findings
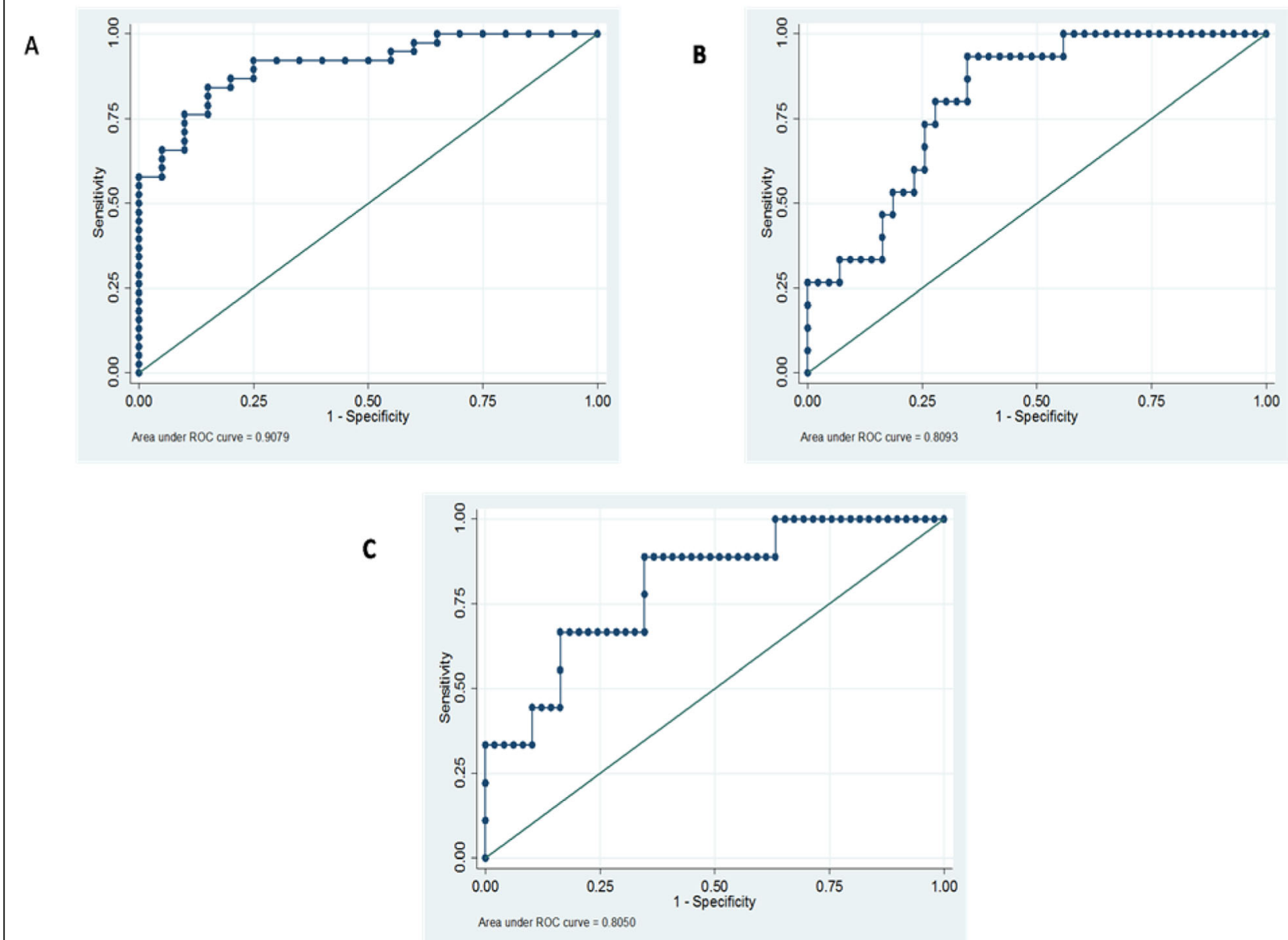

Fig. 2 ROC curve analysis of sFIt-1/PIGF ratio in preeclampsia, which shows the ability of maternal serum sFlt-1/PIGF ratio to (a) differentiate preeclampsia from normal pregnancies; (b) severe PE patients; (c) early-onset PE patients. sFlt-1 has the highest AUC for the diagnosis of PE patients from normal pregnant women 
of other studies which reported association of sFlt1/PlGF ratio with clinical diagnosis of $\mathrm{PE}[4,8,12,18,19]$.

Lim et al. reported higher levels of sFlt-1/PIGF in women with preeclampsia compared to normal controls. Like our study, they reported sFlt-1/PlGF ratio was not significantly different between mild and severe PE patients. Also, the cut-off value of sFlt-1/PlGF was 20.5 with AUC of 0.85 for the prediction of preeclampsia [8]. DE VIVO et al. applied ROC curve analysis for the second-trimester marker values in PE patients. They showed the best diagnostic profile for sFlt-1/PlGF ratio with AUC of 0.92 and the best cut-off of 38.47. They found this marker with the best prediction power for pre-eclampsia with a specificity, a sensitivity, a diagnostic accuracy, a PPV and a NPV of $88.5 \%$, a positive likelihood ratio of 7.7 and a negative likelihood ratio of 0.13 [20]. KUSANOVIC et al. reported the best predictive performance for sFlt1/PlGF ratio with a sensitivity of $100 \%$, specificity of $98-99 \%$, and likelihood ratios for a positive test of $57.6,55.6$ and 89.6 , respectively, for predicting early-onset preeclampsia [21].

Rana et. al. reported sFlt1/PlGF ratio of more than 85 had an association with harmful pregnancy complications and termination of pregnancy in 2 weeks. Moreover, they reported sFlt1/PlGF ratio along with systolic blood pressure (SBP) and proteinuria were a better predictive tool rather than SBP, proteinuria, and uric acid levels [3]. Ohkuchi et al. showed the best diagnostic power of sFlt1/PlGF ratio for both early and late onset PE. They reported a cut-off value of 45 with 97 and 95\% sensitivity and specificity respectively for diagnosis of all preeclampsia and for diagnosis of earlyonset PE (100 and 95\%) [22].

Meanwhile, sFlt1/PlGF ratio could be a useful marker for the differential diagnosis of PE [3]. Hypertension and proteinuria are hallmark symptoms of both preeclampsia and chronic kidney disease (CKD). It is important to differentiate PE from CKD and manage PE efficiently. Rolfo et al. found that serum levels of sFlt1/PlGF ratio significantly increase in PE compared with CKD and controls [23]. Our results support previous studies about the association of high sFlt-1/PIGF ratio with the diagnosis of PE and also severe and early-onset forms of the disease. This marker has the highest sensitivity and specificity for distinguishing PE from normal pregnancies in comparison to severe and early-onset forms of the disease. Using angiogenic biomarkers might lead to screening and faster diagnosis of PE patients and also to preventing them from serious complications. One of the limitations of this study was the sample size. More studies in larger populations and among other ethnicities are required to determine the cost-effectiveness of sFlt-1/PlGF ratio as a biomarker for PE diagnosis. Another limitation was that it was not possible to carry out sampling prior to the patients' diagnosis and samples were taken after PE diagnosis.

\section{Conclusions}

Our data showed that sFlt-1/PIGF ratio has the highest accuracy for the diagnosis of PE patients from normal pregnant women in comparison to its power for the diagnosis of severe or early-onset forms of the disease.

\section{Abbreviations \\ PE: Preeclampsia; PIGF: Placental growth factor; ROC curve: Receiver operating characteristic curve; sFlt-1: Soluble fms-like tyrosine kinase-1 \\ Acknowledgements \\ This work was financially supported by Research Vice-Chancellor of Hormoz- gan University. \\ For Medical Science (HUMS) gratefully acknowledged.}

\section{Authors' contributions}

PN and MR contributed to the study design, initial drafting, and data extraction, developed the search strategy and revised the manuscript. NR, FP and FM critically revised the manuscript. SH and MA provided important intellectual input. All authors approved the final version of the manuscript for publication.

\section{Funding}

The study was funded by a grant from Hormozgan University of Medical Sciences (No. 1-HEC-93-7-8) which allowed all steps of the study to be conducted. These steps were study design, data gathering, analysis and interpretation of data, and the writing of the manuscript.

\section{Availability of data and materials}

The datasets used and/or analyzed during the current study are available from the corresponding author on reasonable request.

\section{Ethics approval and consent to participate}

Informed written consent was obtained from all participants under the protocols approved by.

the Ethical Committee of Hormozgan University of Medical Sciences, Iran. (No. 1-HEC-93-7-8).

Consent for Publication.

Not applicable.

\section{Competing interests}

The authors declare that they have no competing interests.

\section{Author details}

${ }^{1}$ Fertility and Infertility Research Center, Hormozgan University of Medical Sciences, Bandar Abbas, Iran. ${ }^{2}$ Mother and Child Welfare Research Center, Hormozgan University of Medical Sciences, Bandar Abbas, Iran. ${ }^{3}$ Molecular Medicine Research Center, Hormozgan Health Institute, Hormozgan

University of Medical Sciences, Bandar Abbas, Iran. ${ }^{4}$ Department of Biostatics, Faculity of ParaMedicine, Hormozgan University of Medical Sciences, Bandar Abbas, Iran.

Received: 27 October 2019 Accepted: 15 January 2020

Published online: 07 February 2020

\section{References}

1. Practice ACO. Practice bulletin\# 33: diagnosis and management of preeclampsia and eclampsia. Obstet Gynecol. 2002;99(1):159-67.

2. Louwen F, Muschol-Steinmetz C, Reinhard J, Reitter A, Yuan J. A lesson for cancer research: placental microarray gene analysis in preeclampsia. Oncotarget. 2012;3(8):759-73.

3. Rana S, Powe CE, Salahuddin S, Verlohren S, Perschel FH, Levine RJ, et al. Angiogenic factors and the risk of adverse outcomes in women with suspected preeclampsia. Circulation. 2012;125(7):911-9.

4. Verlohren S, Galindo A, Schlembach D, Zeisler H, Herraiz I, Moertl MG, et al. An automated method for the determination of the sFlt-1/PIGF ratio in the assessment of preeclampsia. Am J Obstet Gynecol. 2010;202(2):161. e1-e11.

5. Sitras V, Paulssen R, Grønaas H, Leirvik J, Hanssen T, Vårtun $\AA$, et al. Differential placental gene expression in severe preeclampsia. Placenta. 2009;30(5):424-33. 
6. Park HJ, Kim SH, Jung YW, Shim SS, Kim JY, Cho YK, Farina A, Zanello M, Lee KJ, Cha DH. Screening models using multiple markers for early detection of late-onset preeclampsia in low-risk pregnancy. BMC Pregnancy Childbirth. 2014;14(1):35.

7. Tranquilli $A$, Dekker $G$, Magee $L$, et al. The classification, diagnosis and management of the hypertensive disorders of pregnancy: a revised statement from the ISSHP. Pregnancy Hypertens. 2014:4:97-104.

8. Lim JH, Kim SY, Park SY, Yang JH, Kim MY, Ryu HM. Effective prediction of preeclampsia by a combined ratio of angiogenesis-related factors. Obstet Gynecol. 2008;111(6):1403-9.

9. Stepan $H$, Unversucht $A$, Wessel N, Faber R. Predictive value of maternal angiogenic factors in second trimester pregnancies with abnormal uterine perfusion. Hypertension. 2007;49(4):818-24.

10. Huhn EA, Kreienbuehl A, Hoffmann I, Schoetzau A, Lange S, de Tejada BM Hund M, Hoesli I, Lapaire O. Diagnostic accuracy of different soluble fms-like tyrosine kinase 1 and placental growth factor cut-off values in the assessment of preterm and term preeclampsia: a gestational age matched case-control study. Front Med. 2018:5:325.

11. Powe CE, Levine RJ, Karumanchi SA. Preeclampsia, a disease of the maternal endothelium the role of antiangiogenic factors and implications for later cardiovascular disease. Circulation. 2011;123(24):2856-69.

12. Levine R, Maynard SE, Qian C, Lim K-H, England L, Yu KF, et al. Circulating angiogenic factors and the risk of preeclampsia. N Engl J Med. 2004;350(7):672-83.

13. Thadhani R, Mutter WP, Wolf M, Levine RJ, Taylor RN, Sukhatme VP, et al. First trimester placental growth factor and soluble fms-like tyrosine kinase 1 and risk for preeclampsia. J Clin Endocrinol Metab. 2004;89(2):770-5.

14. Schaarschmidt W, Rana S, Stepan $\mathrm{H}$. The course of angiogenic factors in early-vs. late-onset preeclampsia and HELLP syndrome. J Perinat Med. 2013;41(5):511-6.

15. Kendall RL, Thomas KA. Inhibition of vascular endothelial cell growth factor activity by an endogenously encoded soluble receptor. Proc Natl Acad Sci. 1993:90(22):10705-9.

16. Hertig A, Berkane N, Lefevre G, Toumi K, Marti H-P, Capeau J, et al. Maternal serum sFlt1 concentration is an early and reliable predictive marker of preeclampsia. Clin Chem. 2004;50(9):1702-3.

17. Andraweera $P$, Dekker $G$, Roberts $C$. The vascular endothelial growth factor family in adverse pregnancy outcomes. Hum Reprod Update. 2012;18(4):436-57.

18. Romero R, Nien JK, Espinoza J, Todem D, Fu W, Chung H, et al. A longitudinal study of angiogenic (placental growth factor) and antiangiogenic (soluble endoglin and soluble vascular endothelial growth factor receptor-1) factors in normal pregnancy and patients destined to develop preeclampsia and deliver a small for gestational age neonate. $J$ Matern Fetal Neonatal Med. 2008:21(1):9-23.

19. Sunderii S, Gaziano E, Wothe D, Rogers LC, Sibai B, Karumanchi SA, et al. Automated assays for sVEGF R1 and PIGF as an aid in the diagnosis of preterm preeclampsia: a prospective clinical study. Am J Obstet Gynecol. 2010;202(1):40. e1-7.

20. De Vivo A, Baviera G, Giordano D, Todarello G, Corrado F, D'anna R. Endoglin, PIGF and sFlt-1 as markers for predicting pre-eclampsia. Acta Obstet Gynecol Scand. 2009;87:837-42.

21. Kusanovic JP, Romero R, Chaiworapongsa T, Erez O, Mittal P, Vaisbuch E, et al. A prospective cohort study of the value of maternal plasma concentrations of angiogenic and anti-angiogenic factors in early pregnancy and midtrimester in the identification of patients destined to develop preeclampsia. J Matern Fetal Neonatal Med. 2009:22(11):1021-38.

22. Ohkuchi A, Hirashima C, Suzuki H, Takahashi K, Yoshida M, Matsubara S, et al. Evaluation of a new and automated electrochemiluminescence immunoassay for plasma sFlt-1 and PIGF levels in women with preeclampsia. Hypertens Res. 2010;33(5):422-7.

23. Rolfo A, Attini R, Nuzzo AM, Piazzese A, Parisi S, Ferraresi M, et al. Chronic kidney disease may be differentially diagnosed from preeclampsia by serum biomarkers. Kidney Int. 2013;83(1):177-81.

\section{Publisher's Note}

Springer Nature remains neutral with regard to jurisdictional claims in published maps and institutional affiliations.

Ready to submit your research? Choose BMC and benefit from:

- fast, convenient online submission

- thorough peer review by experienced researchers in your field

- rapid publication on acceptance

- support for research data, including large and complex data types

- gold Open Access which fosters wider collaboration and increased citations

- maximum visibility for your research: over $100 \mathrm{M}$ website views per year

At BMC, research is always in progress.

Learn more biomedcentral.com/submissions 